\title{
Melting and metallization of silica in the cores of gas giants, ice giants and super Earths
}

\author{
S. Mazevet,${ }^{1,2}$ T. Tsuchiya, ${ }^{3}$ T. Taniuchi, ${ }^{3}$ A. Benuzzi-Mounaix,${ }^{4,1}$ and F. Guyot ${ }^{5}$ \\ ${ }^{1}$ LUTH, UMR8102, Observatoire de Paris, CNRS, Université Paris Diderot, \\ 5 place Jules Janssen, 92190 Meudon Cedex France \\ ${ }^{2}$ CEA, DAM, DIF, F91297 Arpajon, France \\ ${ }^{3}$ Geodynamics Research Center, Ehime University, \\ 2-5 Bunkyo-cho, Matsuyama, Ehime 790-8577, Japan \\ 4 LULI, Ecole Polytechnique, CNRS, CEA, UPMC, route de Saclay, 91128 Palaiseau, France \\ ${ }^{5}$ IMPMC, Musum National dHistoire Naturelle,, UMR CNRS 75-90, Sorbonne Universit Paris, France
}

\begin{abstract}
The physical state and properties of silicates at conditions encountered in the cores of gas giants, ice giants and of Earth like exoplanets now discovered with masses up to several times the mass of the Earth remains mostly unknown. Here, we report on theoretical predictions of the properties of silica, $\mathrm{SiO}_{2}$, up to $4 \mathrm{TPa}$ and about $20,000 \mathrm{~K}$ using first principle molecular dynamics simulations based on density functional theory. For conditions found in the Super-Earths and in ice giants, we show that silica remains a poor electrical conductor up to 10 Mbar due to an increase in the Si-O coordination with pressure. For Jupiter and Saturn cores, we find that $\mathrm{MgSiO}_{3}$ silicate has not only dissociated into $\mathrm{MgO}$ and $\mathrm{SiO}_{2}$, as shown in previous studies, but that these two phases have likely differentiated to lead to a core made of liquid $\mathrm{SiO}_{2}$ and solid ( $\left.\mathrm{Mg}, \mathrm{Fe}\right) \mathrm{O}$.
\end{abstract}

Little is known of the properties of silicates at pressures greater than those encountered in the Earth's mantle [1]. Beyond $100 \mathrm{GPa}$, and up to the conditions encountered in Jupiters interior, 4-7 TPa, our current understanding is limited to extrapolations of equations of state of the solid phases only valid at moderately low temperatures (few thousands Kelvins) [2]. On the experimental side, the high-pressures (up to 1TPa) and high-temperatures (up to $50000 \mathrm{~K}$ ) domain has been investigated along the principal Hugoniot using dynamical experiments [35]. Dynamical compression techniques based on quasiisentropic path to reduce heating are in progress, but experimental data for super Earth and Jupiter conditions will remain sparse in the near furture. For years to come, the modeling of planetary interiors will mostly rely on calculations, such as first principles simulations, and in the optimum case, validated on the restricted pressure domain accessible experimentally. In the meantime, the discovery of extrasolar planets several times the mass of the Earth such as CoRo 7b (3 Earth mass) [6, 7] or the modeling of the interior structure and evolution of Jupiter, Saturn, Uranus and Neptune in our solar system all require to elucidate the phase diagram of silicates well beyond a few Mbar [1] .

According to accepted planetary formation scenario 8], silicates are, together with water and iron, the main ingredients of the proto-planet embryos. They can grow up to several times the size of the Earth and then accrete hydrogen and helium up to several times the mass of Jupiter or Saturn. The properties of silicates throughout this trajectory in pressure and temperature space need to be better constrained. If they turn liquid, it potentially impacts dissipation and tidal effects that are keys to understand the stability of planetary systems, the erosion of the core of giant planets or even their luminosity if differentiation leads to a significant release of heat. If they turn metallic in pressure, silicates can significantly contribute to the generation of magnetic fields or play a major role in dissipation effects in terrestrial exoplanets.

In contrast to the iron and water phase diagrams that are better constrained in this regime 10 12], the complex behavior of silicates in density and temperature is currently a challenge for first principle simulations based on Density Functional Theory (DFT). Complex silicates constituting planetary interiors such as $(\mathrm{Mg}, \mathrm{Fe}) \mathrm{SiO}_{3}$ and $(\mathrm{Mg}, \mathrm{Fe})_{2} \mathrm{SiO}_{4}$ cannot be directly simulated due to the large numbers of particles needed. A first approach to the stability and complex chemistry occurring at pressures and temperatures relevant to planetary interiors can be inferred by studying the properties of compounds such as $\mathrm{MgSiO}_{3}$, a major silicate endmember in the deep Earth interior and its dissociation products $\mathrm{MgO}$, and $\mathrm{SiO}_{2}[2]$.

Using lattice dynamics, Umemoto et al. 2] established the stability of $\mathrm{MgSiO}_{3}$ (Fig (1) up to pressures representative of Jupiter's interior. Within the quasi-harmonic approximation, they showed that $\mathrm{CaIrO}_{3}$ type $\mathrm{MgSiO}_{3}$, the silicate stable at the terrestrial core-mantle boundary, cannot exist beyond $1 \mathrm{TPa}$ and probably melts at temperatures varying between $5000 \mathrm{~K}$ and $10,000 \mathrm{~K}$ in this pressure range. As shown in Fig 1, this implies that $\mathrm{MgSiO}_{3}$ may remain stable at conditions encountered in Uranus and Neptune but that it would dissociate into $\mathrm{MgO}$ and $\mathrm{SiO}_{2}$ in Saturn and Jupiter. As the quasiharmonic approximation tends to overestimate the melting temperature, whether $\mathrm{MgSiO}_{3}$ is in a liquid or a solid state at conditions found in Uranus and Neptune cores remains an open question. Its answer necessitates to go beyond the quasi-harmonic approximation by including anharmonic effects using molecular dynamics. As a first 


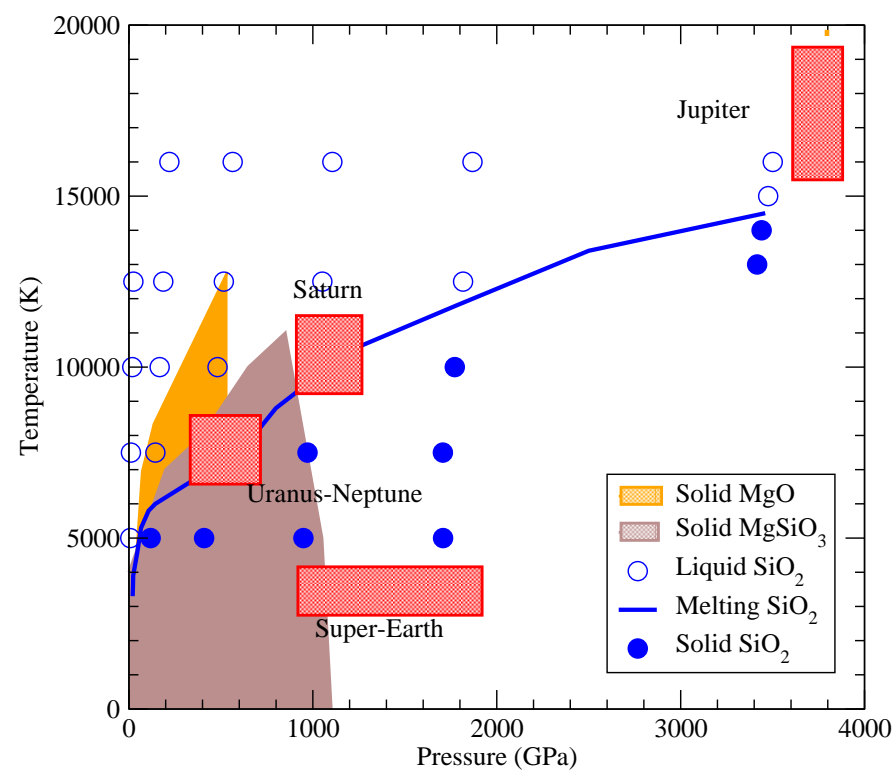

FIG. 1: Phase diagram of $\mathrm{MgSiO}_{3}, \mathrm{SiO}_{2}, \mathrm{MgO}$. (Orange) stability of $\mathrm{MgSiO}_{3}$ in pressure and temperature. (Brown) Solid $\mathrm{MgO}$. (Blue) $\mathrm{SiO}_{2}$ high-pressure melting curve. (Filled blue dots) calculated solid $\mathrm{SiO} 2$, (open blue dots) calculated liquid $\mathrm{SiO} 2$

step toward a full ab initio phase diagram of silicates in the pressure-temperature range relevant to planetary modeling, we calculated the physical properties of silica, $\mathrm{SiO}_{2}$, using DFT based molecular dynamics simulations (DFT-MD) for the high pressure melting curve and linear response theory for the electrical properties.

For the high-pressure melting curve, we performed twophase molecular dynamics simulations up to $4 \mathrm{TPa}$ as this pressure range is representative of Jupiter's interior. We used specifically designed pseudo-potentials with $2 \mathrm{~s}$ and $2 \mathrm{p}$ shells included as valence states to reach the highest pressures investigated here. This suit of pseudopotentials were validated against all-electron calculations for the relevant range of pressure 18] Our two-phase MD simulations were performed using the pwscf code [19] for electronic structure with an original implementation of the temperature constant MD solver, where the temperature $T$ was controlled to keep constant values by the kinetic energy scaling method[20].

Two phases, both with the melt and crystalline structures, were placed in a computational box. Here, both phases contained $24 \mathrm{SiO}_{2}$ units (72 atoms), and the computational box contained 48 units (144 atoms) total. The simulation procedure was as follows: the crystalline structure at each pressure was first equilibrated at 2000 $\mathrm{K}$ for a period of $1 \mathrm{ps}\left(10^{-12} \mathrm{~s}\right)$. The liquid phase at each pressure were obtained by equilibrating at $20000 \mathrm{~K}$ for a period of $1 \mathrm{ps}$. The solid and liquid structures were put

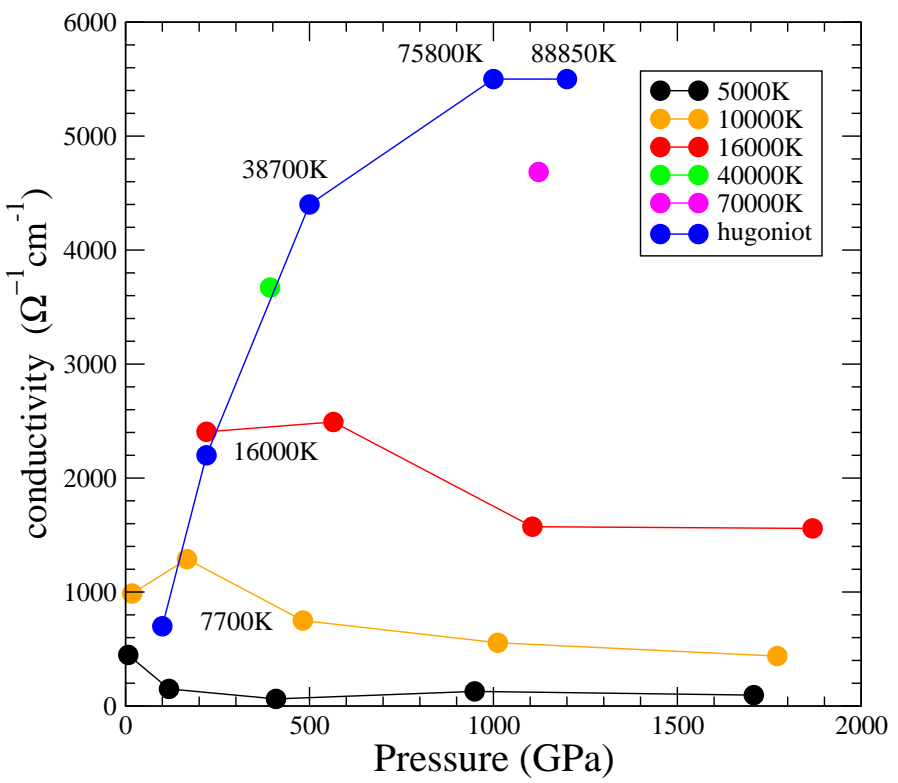

FIG. 2: Variation of the electrical conductivity in pressure along various isotherms and at conditions relevant to planetary modeling. Solid lines are guides to the eyes.

together with a small spacing in a simulation box, letting the lower part of the box be solid while keeping the upper part liquid. We thermalized the liquid cells for 50 time steps at target temperatures before starting two phasessimulations. In our simulations, the solid and liquid cells were connected at the (001) plane of solid. Starting from these configurations, the MD simulations evolve into a single phase. For temperatures above the melting temperature, $T_{m}$, the cell will turn liquid, whereas the cell will solidify in a vitreous state at temperature below $T_{m}$. The simulations were typically performed for 10000 time steps (10 ps). $T_{m}$ at pressures below $160 \mathrm{GPa}$ were obtained from reference [20].

In the $\mathrm{SiO}_{2}$ compound, several phases have been identified as stable at ultra-high-pressures including an hexagonal $\mathrm{Fe}_{2} \mathrm{P}$-type structure at $1 \mathrm{TPa}$, beyond a cotunnitetype phase [12]. These solid phases were used in twophases simulations to calculate the melting temperature. This led to different slopes in the melting curve and to discontinuities observable in Fig 1. From 0.1TPa and up to $1 \mathrm{TPa}$, Fig 1 shows that the melting temperature increases up to $10,000 \mathrm{~K}$. This region corresponds to conditions anticipated at the cores of Uranus, Neptune, and Saturn [1].

Fig[1 also shows that for these three planets, a core constituted of pure silica would be molten in the core as the pressure and temperature conditions anticipated lay above the calculated high pressure-melting curve. The situation is somewhat tempered in the cases of Uranus and Neptune as the quasi-harmonic approximation esti- 
mates that $\mathrm{CalrO}_{3}$-type $\mathrm{MgSiO}_{3}$ remains stable at these conditions [2]. As anharmonic effects may lead to a lower melting temperature and a smaller $\mathrm{MgSiO}_{3}$ stability domain of solid phases in pressure and temperature, the calculations shown here suggest that the cores of Uranus and Neptune-type planets would also be constituted of a significant fraction of melted silica if $\mathrm{MgSiO}_{3}$ is shown to be dissociated at these conditions.

The situation for Jupiter and Saturn's cores is clearer. Fig 1 shows that silica is molten at these conditions. In this pressure-temperature range, $\mathrm{CalrO}_{3}$-type $\mathrm{MgSiO}_{3}$ is not stable and is predicted to dissociate into $\mathrm{MgO}$ and $\mathrm{SiO}_{2}$ within the quasi-harmonic approximation. We also note that DFT-MD simulations [13] show that the $\mathrm{MgO}$ melting temperature increases with pressure much faster than either those of $\mathrm{MgSiO}_{3}$ or $\mathrm{SiO}_{2}$ up to $0.5 \mathrm{TPa}$. An extrapolation of the $\mathrm{MgO}$ high-pressure melting curve up to the conditions of Saturn's core, 1TPa, suggests that it remains solid and probably in the B2 phase. This indicates that during its evolution, Saturn's silicates are probably dissociated into two components, solid $\mathrm{MgO}$ and liquid $\mathrm{SiO}_{2}$. Considering the rather close densities of these two constituents at a pressure of $0.5 \mathrm{TPa}(7 \mathrm{~g} / \mathrm{cm} 3$ and $7.2 \mathrm{~g} / \mathrm{cm} 3$ respectively) and without direct calculations of the $\mathrm{MgO}$ properties at $1 \mathrm{TPa}$, the calculations shown here indicate that Saturn's silicates could turn in either a $\mathrm{MgO}$ shell surrounding a silicate liquid core or conversely a $\mathrm{MgO}$ core surrounded by a layer of liquid silica. The presence of iron, likely preferentially dissolved in $\mathrm{MgO}$ would increase the density of this latter phase and favor a core made of a central $(\mathrm{Mg}, \mathrm{Fe}) \mathrm{O}$ surrounded by molten $\mathrm{SiO}_{2}$. These considerations also apply to Jupiters core. We note, however, that it exists at the moment a wide disparity between models regarding the conditions at Jupiters core with predictions for temperatures at the envelopp-core boundary ranging from 15,000 to $20,000 \mathrm{~K}$ [1].

Fig 1 also shows that silica remains in a solid state in Earth like exoplanets up to several times the Earth mass. Whether silica turns metallic at these conditions is a second important issue that we investigated using linear response theory. To obtain the transport properties, we performed a set of simulations starting from the quartz alpha phase with 108 atoms in the simulation cell. We used the isokinetic ensemble and performed the calculations with the Abinit electronic structure code 21]. We checked that the simulation relaxes into the proper phase at a given pressure-temperature condition by calculating the Si-O coordination number. From equilibrated trajectories, we performed Kubo-Greenwood calculations on several snapshots to obtain the transport properties. Within this formulation 22], the real part of the optical conductivity is obtained by using the eigenvalues and eigenfunctions obtained from the diagonalization of the Kohn-Sham Hamiltonian. We used 5 snapshots and a 43 k-points grid as given by the Monkhorst-Pack scheme 23]

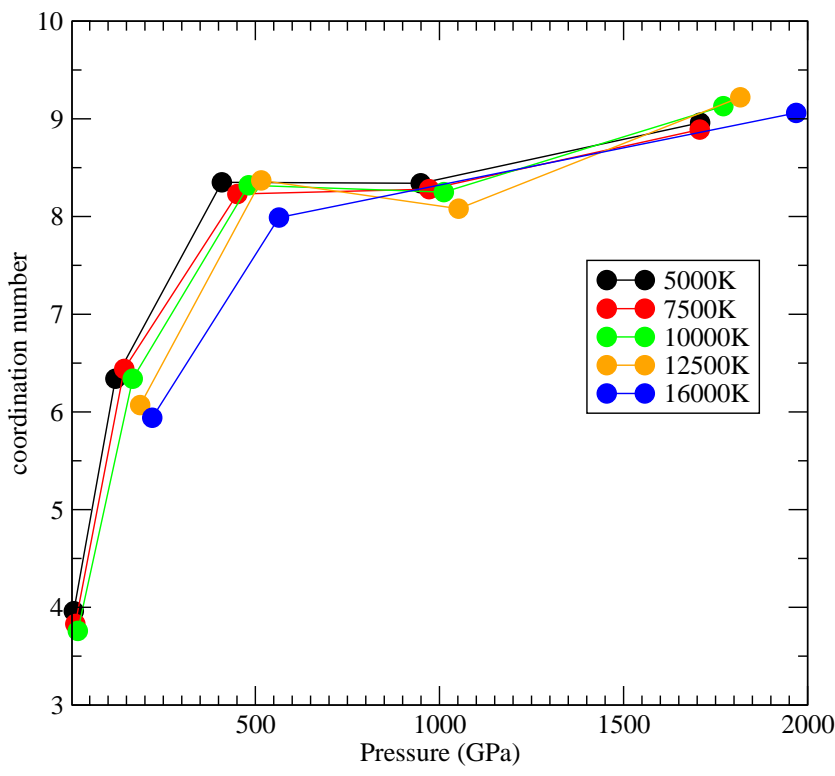

FIG. 3: Variation of the coordination number (CON) in pressure along five isotherms.

to insure convergence of the optical conductivity in particle numbers, k-points, and number of snapshots. We used 800 bands to insure convergence of the optical conductivity in photon frequency well beyond $10 \mathrm{eV}$. The direct current (DC) conductivity plotted in Fig2 is obtained by taking the limit of the optical conductivity at zero frequency.

Fig 2 shows DC conductivity calculated using supercells equilibrated along several isotherms as indicated in Fig 1 We find that the DC conductivity increases as temperature increases along the principal Hugoniot (i.e. the final states reached in a shock experiment starting from normal conditions) to reach a plateau at 5500 $\mathrm{ohm}^{-1} \mathrm{~cm}^{-1}$, concomitantly with the disappearance of Si-O bonding [14]. This is in very good agreement with shock wave experiments [3]. Surprisingly, Fig 2also shows that no non-metal-metal transition is observed in silica as pressures increase along an isotherm. The DC electrical conductivity actually does not increase with pressure along a given isotherm but rather decreases. This is unexpected since many insulators undergo a pressure-induced metallization at relatively low temperatures [15, 16]. The origin of this effect can be traced back to the change in the $\mathrm{Si}-\mathrm{O}$ coordination with pressure and the corresponding variation of the electronic structure.

It is well documented that the $\mathrm{Si}-\mathrm{O}$ coordination increases from 4 in alpha-quartz to 9 in the cotunnite and $\mathrm{Fe}_{2} \mathrm{P}$ phases 12]. Fig 3 shows the values of the coordi- 


\section{DOS Occupied DOS}

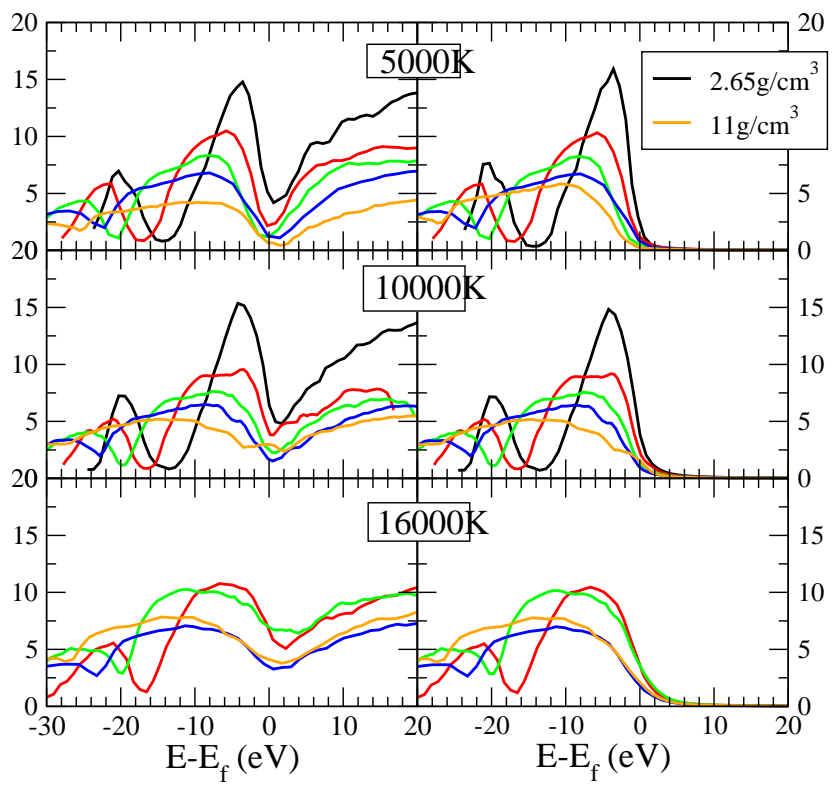

FIG. 4: Variation of the density of states (DOS) and occupied density of states in temperature and pressure. Each raw corresponds to an isotherm as indicated in the figure. The densities are (black) $2.65 \mathrm{~g} / \mathrm{cm}^{3}$, (red) $5 \mathrm{~g} / \mathrm{cm}^{3}$, (green) $7 \mathrm{~g} / \mathrm{cm}^{3}$, (blue) $9 \mathrm{~g} / \mathrm{cm}^{3}$, (orange) $11 \mathrm{~g} / \mathrm{cm}^{3}$.

nation number $(\mathrm{CON})$ obtained by integrating the $\mathrm{Si}-\mathrm{O}$ correlation function up to $r_{c u t}=4.5 \mathrm{a}_{B}$ at the lowest densities and up to $r_{c u t}=4 \mathrm{a}_{B}$ at $9 \mathrm{~g} / \mathrm{cm}^{3}$ and $11 \mathrm{~g} / \mathrm{cm}^{3}$. These cutoff values correspond to the minimum in the Si-O pair correlation function following the first peak. Fig 3 shows that the Si-O CON has a similar behavior in either the solid or the liquid at these temperatures with values corresponding to the ones obtained previously for the $\mathrm{Fe}_{2} \mathrm{P}$-type structure 12]. Fig 3 also shows that temperature has little effect on the Si-O CON at these conditions, although a decrease in the Si-O CON in temperature is still noticeable at each density. The increase in the $\mathrm{Si}-\mathrm{O} \mathrm{CON}$ with pressure corresponds to a drastic modification of the electronic structure.

Fig 4 shows the density of states (DOS) and occupied density of states (occupied DOS) obtained at the densities corresponding to the conductivities given in Fig[2] and for three representative isotherms. Fig 4 shows that silica behaves as a semi-metal at low density and moderate temperatures with a pseudo-gap clearly visible around the Fermi energy. Along an isotherm, the increase in CON corresponds to the opening of a pseudo-gap and a decrease in the density of state around the Fermi en- ergy. The latter is clearly visible in the occupied DOS. Conversely, comparing the DOS and occupied DOS in between isotherms at a given density shows that temperature has the opposite effect on the electronic structure. This suggests that pressure along an isotherm tends to increase the localization of the electron on each atom of the Si-O bond, i.e. implicitly increasing its ionic character. It also shows that the effect of temperature is more involved than a simple temperature activated process across a gap fixed at a given density. As temperature decreases the $\mathrm{Si}-\mathrm{O} \mathrm{CON}$ increases at a given density, the DOS and occupied DOS are modified accordingly and lead to smaller conductivities as shown in Fig 2,

This behavior of silica leads to a significant correction of previous estimates of the electrical conductivity by an order of magnitude 2]. We find that the DC electrical conductivity at conditions relevant to the deepest mantles of super-Earths is of the order of 200-300 ohm $-1 \mathrm{~cm}-1$. Contrary to previous speculations, this suggests that silica will not contribute significantly to either magnetic field generation in Earth like exoplanets up to several times the Earth mass. The DC electrical conductivity of silica starts to be comparable to the one of a poor metal (greater than $1000 \mathrm{ohm}^{-1} \mathrm{~cm}^{-1}$ ) above 7000-8000K.

We established here new equation of state data for silica needed to build self-consistent planetary models of ice giants, gas giants, and terrestrial exoplanets . Contrary to previous estimates, we find that silica remains a poor metal at extreme pressures with little contribution to transport properties and magnetic field in terrestrial exoplanets. The molten state of $\mathrm{SiO} 2$ at conditions deep in Jupiter and Saturn, and possibly in Uranus and Neptune, suggests that structure and evolution models of such bodies may need to account for a liquid state in their cores. This is also the case for standard tidal models that consider dissipation as mostly occurring in a solid core made of silicates, water and iron [17].

This work is supported in part by the French Agence National de la Recherche under contract PLANETLAB ANR-12-BS04-0015.

[1] I. Baraffe et al., Rep. Prog. Phys. 73, 016901 (2010).

[2] K. Umemoto et al., Science 311, 983 (2006).

[3] D. Hicks et al., Phys.Rev. Lett. 97, 025502 (2006).

[4] M. Knudson et al., Phys.Rev.Lett. 103, 225501 (2009).

[5] D.K Spaulding et al, Phys. Rev. Lett. 108, 065701 (2012).

[6] E. J. Rivera et al., Astrophys. J. 634, 625 (2005).

[7] D. Queloz et al., A\&A 506 303-319 (2009).

[8] T. Guillot, Science 286, 72 (1999).

[9] J. Bouchet et al., Phys. Rev. B 87094102 (2013).

[10] R. Redmer et al., Icarus 211798 (2011).

[11] L. Stixrude, Phys. Rev. Lett 108055505 (2012).

[12] T. Tsuchiya et al., PNAS 108, 1252 (2011). 
[13] A.B. Belonoshko et al., Phys. Rev. B 81: 054110 (2010).

[14] Y. Laudernet et al., Phys. Rev. B 70165108 (2004).

[15] N.F. Mott, Philos. Mag. 6, 287 (1961).

[16] M.I. Eremets and I.A. Troyan, Nature Materials 10, 927 (2011).

[17] F. Remus et al., A\&A 544 A132 (2012).

[18] F. Jollet et al., arXiv:1309.7274 (2013).
[19] P. Giannozzi et al., J. Phys. Cond. Mat. 21, 395502 (2009).

[20] Y. Usui and T. Tsuchiya, J. Earth Sci. 21, 801 (2010).

[21] X. Gonze et al. Comp. Phys. Comm 180, 2582.(2009).

[22] S. Mazevet et al., HEDP 6, 84-88. (2010).

[23] H. J. Monkhorst et al. Phys. Rev. B 13, 5188 (1976). 